\title{
INCORPORACIÓN DE PREDICCIONES CLIMÁTICAS Y MEDIDAS DE SENSORES DE HUMEDAD DE SUELO EN EL RIEGO DE PRECISIÓN
}

\author{
Flores Cayuela, C. (1) (P), Camacho Poyato, E., Montesinos Barrios, $P$.
}

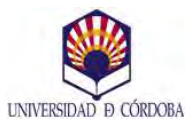

${ }^{1}$ Dpto. Agronomía, ETSIAM, Universidad de Córdoba. Ed. Leonardo da Vinci, Campus de Rabanales,14071 Córdoba, g02flcac@uco.es

\section{Introducción}

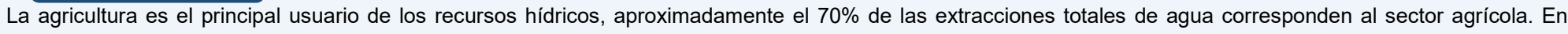

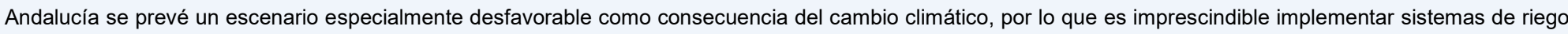

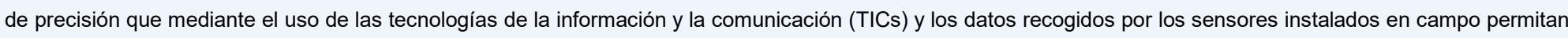

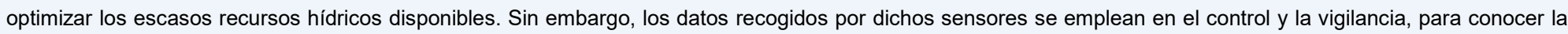

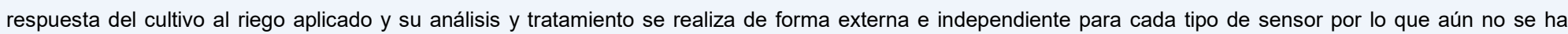
extraído todo el potencial de esta tecnología.

\section{Objetivos. Implantar un sistema de riego de precisión en cultivos leñosos basado en predicciones de la evapotranspiración de referencia (ETo) obtenidas a} partir de predicciones meteorológicas y en el análisis de los datos recogidos por los sensores de humedad de suelo instalados en campo

\section{Materiales y métodos}

\section{* 1) Esquema de funcionamiento}

La Fig.1 muestra la información usada por el modelo para determinar la programación y momento óptimo de riego.

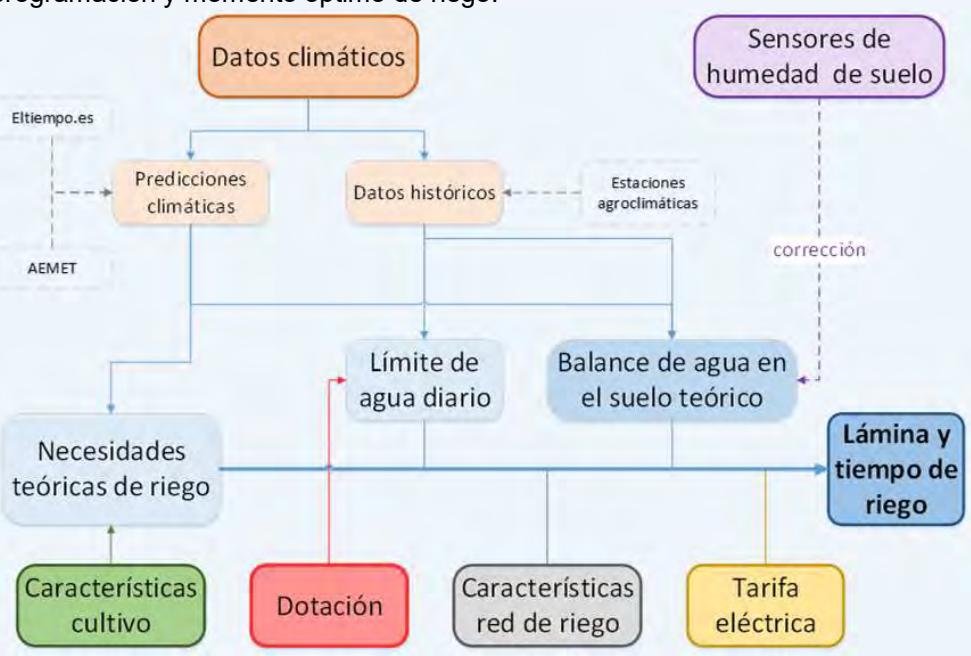

Figura 1 - Diagrama de flujo

\section{2) Uso de los sensores}

$\mathrm{Dr}_{\text {Teórico }}$

$\operatorname{Dr}_{\mathrm{i}}=\mathrm{Dr}_{\mathrm{i}-1}-\mathrm{Pef}_{\mathrm{i}}-\left(\mathrm{NRb}_{\mathrm{i}}{ }^{*} \mathrm{IE}\right)+\left(\mathrm{ETo}_{\mathrm{i}}{ }^{*} \mathrm{Kc}_{\mathrm{i}}{ }^{*} \mathrm{Ks}_{\mathrm{i}-1}\right)$

Dr=Nivel de agotamiento $(\mathrm{mm}) \quad \mathrm{Kc}=$ Coeficiente de cultivo Pef=Precipitación efectiva $(\mathrm{mm}) \quad \mathrm{Kc}=$ Coeficiente de cultivo $\mathrm{NRb}=$ Lámina de riego bruta(n

Eto=Evapotrans
referencia $(\mathrm{mm})$

\section{3) Decisión de riego}
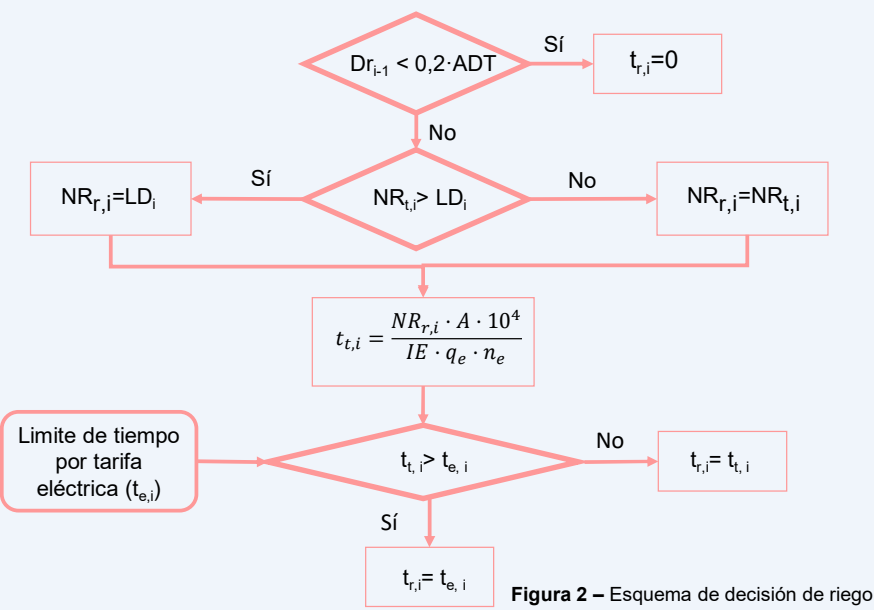

ADT $=$ Total de agua disponible en el suelo

$t_{r, i}=$ Tiempo de riego recomendado $(h)$ $0,2 \cdot A D T=$ Nivel de agotamiento permisible (NAP) $A=$ Área asociada al sector (ha) $N R_{r, i}=$ necesidades de riego reales $(\mathrm{mm}) \quad \mathrm{q}_{\mathrm{e}}=$ Caudal del emisor $(\mathrm{l} / \mathrm{h})$ $L D_{i}=$ Límite de dotación diario $(\mathrm{mm})$ $\mathrm{t}_{\mathrm{t}, \mathrm{i}}=$ Tiempo teórico de riego requerido $(\mathrm{h}) \quad \mathrm{IE}=$ Eficiencia del riego

\section{Resultados}

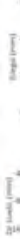

,

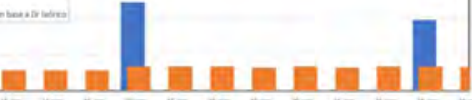

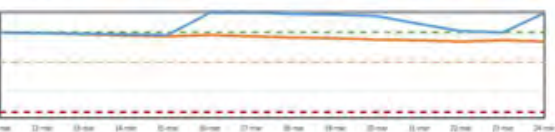

La metodología propuesta se ha aplicado a la programación de riegos de precisión en una finca de naranjo durante la campaña 2019, comparando esta programación con la basada en el cálculo teórico del déficit de humedad en el suelo. La figura 1 muestra la diferencia entre en $\operatorname{Dr}_{\text {teórico }}$ y el $\operatorname{Dr}_{i}$ corregido con los valores de las sondas y su efecto en la programación del riego. Aunque el $\mathrm{Dr}_{\text {teórico }}$ baja levemente con el riego real aplicado por el agricultor, sigue siendo superior al NAP por lo que se programan riegos para todos los días, en cambio al incorporar los datos registrados por las sondas tras los riegos se comprueba que la disponibilidad de agua en e suelo es suficiente para cubrir las necesidades del cultivo los próximos 7 días.

\section{Conclusiones}

La metodología desarrollada permite optimizar el momento de riego, evitando que se realicen riegos cuando el contenido de humedad en el suelo es suficiente para cubrir las necesidades de la planta, con el consiguiente ahorro tanto de agua como de energía.

\section{AGRADECIMIENTOS:}

Este trabajo forma parte del proyecto HUELLA DEL AGUA EN EL SECTOR ECOLÓGICO ANDALUZ, cofinanciado por la Junta de Andalucía y la Unión Europea a través del FEADER 